iment leads to the stabilization of the processes of peroxidation against the increase of antioxidant system activity.

Key words: remaxol, carbon tetrachloride, biological membranes lipid peroxidation, products of peroxidation (lipid hydroperoxides, diene conjugates, malonic dialdehyde), antioxidant system.

In connection with the peculiarities of molecular mechanisms of action of carbon tetrachloride on subcellular membranes, the study of the biological action of hepatotropic poison is of interest as a model of the molecular pathology of membrane structures. Carbon tetrachloride causes a dose-dependent liver damage, in addition to exerting a toxic effect on blood cells, connective tissue, morphological and functional state of a number of organs and systems. According to modern concepts, a significant role in the development of pathology in toxic liver play of free radical reactions. Reactive oxygen species cause increased peroxidation of lipids of cellular membranes, contributing to the imbalance between Pro - and antioxidant systems with the subsequent formation of oxidative stress in warm-blooded body, which is pathogenetically justified the appointment of drugs with antioxidant and membrane-protective effect. These properties were detected in medicines containing succinic acid, having broad spectrum of pharmacological activity.

Materials and methods. In experimental conditions the possibility to correct free radical lipid oxidation of rats' organism membranes was studied with the introduction of the succinate containing drug called remaxol (Polysan, St. Petersburg). The animals were divided into 4 groups and each of them had 20 rats: intact animals which were held in standard conditions of vivarium; the control group in which rats intake of the carbon tetrachloride during 3 days daily; the experimental group in which before the introduction of carbon tetrachloride animals had a daily intra-abdominal intake of the remaxol in a dose of $50 \mathrm{mg} / \mathrm{kg}$; the experimental group in which before the introduction of carbon tetrachloride animals had a daily intra-abdominal intake of the remaxol in a dose of $100 \mathrm{mg} / \mathrm{kg}$. The intensity of peroxidation processes was assessed by examining the contents of hydroperoxides lipids, diene conjugates, malonic dialdehyde and the main components of the antioxidant system, (ceruloplasmin, vitamin E) in the plasma of blood animals. The results obtained were subjected to statistical analysis with calculation of parametric criteria Student.

It was found out that in the blood of experimental animals a daily the introduction of carbon tetrachloride during 3 days contributes to the increase of lipid hydroperoxides level (by $20-24 \%$ ), of diene conjugate (by 17 - 19\%), and of malonic dialdehyde (by 51-59\%) against the decrease of antioxidant system activity in the blood of intact animals. The introduction of the succinate containing drug to rats in the conditions of oxidative stress contributes to the reliable decrease in the blood of lipid hydroperoxides by $7-12 \%$, of diene conjugates-by $7-11 \%$, and of malonic dialdehyde by $13-24 \%$ in comparison with the rats of the control group. While analyzing the effect of the succinate containing drug on the activity of the components of antioxidant system it was shown that the level of ceruloplasmin in the blood of animals was reliably higher by $9-24 \%$, of vitamin E by $12-21 \%$, of catalase by $13-24 \%$ in comparison with the same parameters of the rats of the control group.

So, the application of the succinate containing drug called remaxol in the conditions of introduction of carbon tetrachloride of the organism of animals under experiment leads to the stabilization of the processes of peroxidation against the increase of antioxidant system activity.

\title{
REFERENCES
}

Dorovskikh V.A., Borodin E.A., Lee O.N., Simonova N.V., Starberg M.A., Dorovskikh Yu.V. Comparative efficiency of different antioxidants during cold exposure and overheating. Blagoveshchensk; 2016 (in russian).

Dorovskikh V.A., Borodin E.A., Shtarberg M.A., Shtarberg S.A., Egorov K.E. Phospholipids as antiatherosclerotic drugs. In book: Lipoproteins and atherosclerosis. Abstracts of the Symposium devoted to the 110 anniversary from the birthday of academician N.N. Anichkov «Lipoproteins and atherosclerosis». Moscow; 1995: 41-46 (in russian).

Dorovskikh V.A., Tseluyko S.S., Simonova N.V., Anokhina R.A. In the world of antioxidants. Blagoveshchensk, 2012 (in russian).

Landyshev Ju.S., Dorovskikh V.A., Tseluyko S.S., Lazutkina E.L., Tkacheva S.I., Chaplenko T.N. Bronchial asthma. Blagoveshchensk, 2010 (in russian).

Landyshev Ju.S., Dorovskikh V.A., Chaplenko T.N. Drug Allergy. St-Petersburg, 2010 (in russian).

Simonova I.V., Dorovskikh V.A., Simonova N.V., Shtarberg M.A. Non-specific preventive measures against respiratory diseases of nursery age children. Far Eastern Medical Journal 2009; 3:56-58 (in russian).

Simonova N.V., Dorovskikh V.A., Shtarberg M.A. Effect of adaptogens of plant origin on the intensity of the processes of peroxidation of lipids of membranes under conditions of ultraviolet irradiation. Far Eastern Medical Journal 2010; 2:112-115 (in russian).

Simonova N.V., Dorovskikh V.A., Simonova N.P. Ultraviolet radiation and oxidative stress. The possibility of phitocorrection. Blagoveshchensk, 2014 (in russian).

Simonova N.V., Dorovskikh V.A., Li O.N., Shtarberg M.A., Simonova N.P. Tincture of medicinal plants and oxidative stress in the conditions of cold influence. Bulletin physiology and pathology of respiration 2013; 48:76-80 (in russian).

DOI 10.22448/AMJ.2017.3.28-29

OBSERVATION ON THE THERAPEUTIC EFFECT OF BAILING CAPSULE ON HASHIMOTO ‘S THYROIDITIS

\section{DULikun, ZHOUHaili}

Heilongjiang University of Traditional Chinese Medicine Harbin City, Heilongjiang Province, China

Abstract:Objective:to investigate the clinical efficacy of Bailing Capsule in Hashimoto thyroiditis (HT). Methods:A total of 100 patients who come from the First Affiliated Hospital of Heilongjiang University of Traditional Chines Med- 
icine in 2016 aged 20 to 65 years were randomly divided into control group $(n=54)$ and observation group $(n=$ 46). The control group routine treatment and low iodine diet, the observation group in the conventional treatment based on Bailing capsule interventional treatment,serum levels of free triiodothyronine (FT3), free thyroxine (FT4), thyrotropin (TSH), thyroglobulin antibody (TGAb),thyroid per-oxidase antibody(TPOAb), were measured before and 6 months after treatment, while thyroid ultrasonography.The data were analyzed by SPSS17.0 statistical software. The data were expressed as mean \pm standard deviation by the $t$ test and Thex 2 test was used to count data rate (\%). $P<0.05$ for the difference was statistically learning meaning. Results: The TGAb and TPOAb titers of the treatment group were significantly lower than those before and after treatment $(P<0.01)$. The thickness of the isthmus in the treatment group was significantly smaller than that before treatment $(P<0.01)$. Conclusion: Bailing capsule can reduce the TGAb, TPOAb titer, improve the autoimmune response, and further delay the occurrence of hypothyroidism.

Key words: Bailing capsule; Hashimoto's thyroiditis; Antibody

$\mathrm{HT}$ is a group of diseases caused by autoimmune thyroid dysfunction, clinical manifestations of thyroid enlargement, $50 \%$ with clinical hypothyroidism, treatment is currently the most difficult problem of the disease.Over the years people use selenium yeast can reduce the titer of thyroid antibodies, but the effect is poor.The main components of Bailingcapsulewere fermented Cordyceps sinensis powder (CS-C-Q80), modern pharmacological detection of Cordyceps sinensis has a wide rangeofimmuneregulation, thisstudy of itstreatment of 100 cases ofHTpatients, theeffectisobvious. Thereportisasfollows.

\section{Materials and methods}

1.1General informationA total of 100 patients ( 35 males and 65 females, aged 20 to 65 years) with a duration of less than 3 months were selected. There were no symptoms of discomfort in the treatment of 100 patients who were diagnosed with endocrine clinics in the First Affiliated Hospital of Heilongjiang University of Traditional Chinese Medicine in 2016..Randomly divided into control group ( 54 cases ) and observation group ( 46 cases), all patients had normal thyroid function, but both TGAb,TPOAb increased.The general data of the control group and the treatment group were not statistically significant, comparable.

1.2Treatment Control group routine treatment and low iodine diet, do not take other immunomodulators. The observation group on the basis of conventional treatment to giveBailingcapsule,2/time,3times/ day, continuous medication for 6 months. Fasting blood was extracted before and after treatment, thyroid function and antibody index were measured, and the size of thyroid before and after treatment was measured by color Ultrasonic.

1.3Diagnostic criteriaRefer to Morimoto land standard: (1) Diffuse and hard goiter; (2) Serum anti-thyroid autoantibody positive; (3) Pathological histology of HT. All can have a clear diagnosis, with (1), (2) or (1), (3) items can also be diagnosed.

1.4Statistical processingSPSS17.0statistical softwareforstatisticalanalysis, measurement datawiththemean \pm standarddeviationsaid, usingttest, count datarate(\%)said, usingx 2 test, $P<0.05$ forthedifferencewasstatisticallysignificantsignificance.

2 results

2.1 Comparison of thyroid function between the two groups before and after treatment There was no significant difference in the trend of thyroid function FT3, FT4 and TSH before and after treatment between the two groups $(P>0.05)$. But TGAb and TPOAb were significantly lower than those before treatment $(P<0.01)$.The observation group of the two groups was more obvious than the control group $(P<0.01)$.

2.2 Comparison of thyroid size between the two groups before and after treatment There were no significant differences between the two groups before and after treatment $(P>0.05)$. The thickness of isthmus in the observation group was significantly smaller than that before treatment, the difference was statistically significant $(P<0.01)$. The thickness of isthmus in the control group was slightly smaller than that before treatment, the difference was not statistically significant $(P>0.05)$.

\section{3 conclusion}

The results show that Bailing capsule can significantly reduce the HT patients TGAb, TPOAb titer, play a role in reducing the immune response of patients, the patient's condition can be effective and sustained control, worthy of clinical application. The results of this study found that Bailing capsule only to reduce the thickness of the thyroid gland, and on the thyroid function, before and after the left and right and the upper and lower diameter no significant effect may be due to short observation time, thyroid scaling still need some time to show it, so the conclusion needs large sample, long research argument.

The author's e-mail:zhl19930210@163.com

Mailing address:Heilongjiang University of Traditional Chinese Medicine Graduate School,No.24,Heping Road,Xiangfang District,Harbin City, Heilongjiang Province. 